\title{
Clinical Study on the Aerosol Inhalation of Budesonide in Treatment of Acute Exacerbation of COPD
}

\author{
Huifeng Duan* \\ TCM hospital of Xinmin city, Xinmin, Liaoning 110300, China
}

\begin{abstract}
Objective: To observe the clinical effect and adverse reaction of aerosol inhalation of budesonide in treatment of acute exacerbation of COPD. 110 cases of patients with acute exacerbation of chronic obstructive pulmonary disease, who were treated in our department from January 2012 to January 2014, were selected as research objects (69 males, 41 females). All the patients were divided into control group and observation group. The two groups were treated routinely, in addition, the control group was treated with vein administration of methylprednisolone, the observation group received aerosol inhalation of budesonide, observed the clinical effect. Result: The clinical effect of observation group is better and the adverse reaction rate is clearly lower. Conclusion: Comparing with the control group which is treated with vein administration of methylprednisolone on the basis of routine treatment, the effect of observation group which is treated with aerosol inhalation of budesonide is extremely precise and its adverse reaction is also relatively less. Meanwhile, it has a higher security and its clinical effect is worth applying.
\end{abstract}

\author{
KEYWORDS \\ Budesonide \\ Aerosol \\ Acute exacerbation \\ COPD
}

\section{Introduction}

Chronic obstructive pulmonary disease (COPD) is a common chronic lung disease in clinical and its characteristic is airflow obstruction. Acute exacerbation of chronic obstructive pulmonary disease (AECOPD) refers to a clinical manifestation of sharp deterioration of cough, expectoration, inhalation or asthmatic in short time of chronic obstructive pulmonary disease. The sputum volume increases obviously and the property of sputum

Copyright @ 2016 Huifeng Duan

doi: 10.18686/aem.v5i3.13

Received: August 6, 2016; Accepted: September 4, 2016; Published online: September 20, 2016

This is an open-access article distributed under the terms of the Creative Commons Attribution Unported License (http://creativecommons.org/ licenses/by-nc/4.0/), which permits unrestricted use, distribution, and reproduction in any medium, provided the original work is properly cited.

${ }^{\star}$ Corresponding author: TCM hospital of Xinmin city, Xinmin, Liaoning 110300, China. E-mail: duanhuif@sina.com is mucopurulent that mean the inflammation aggravates obviously [1]. Acute exacerbation of COPD can cause serious damage to the pulmonary function and may develop into pulmonary heart disease, leads to respiratory failure and becomes a serious threat to the patients' safety [2]. In recent years, studies have shown that glucocorticoid can reduce the clinical symptoms of patients suffering from acute exacerbation of COPD and speed up the recovery of pulmonary function [3]. In this paper, we observe the clinical effect of 55 cases of patients treated with aerosol inhalation of budesonide. The report is as follows.

\section{Objects and methods}

\subsection{General information}

110 cases of patients with acute exacerbation of chronic obstructive pulmonary disease, who received treatment in our hospital from January 2012 to January 2014, were selected as research objects (69 males, 41 females). The age was within the range of 50 to 83 years old and the average 
was 69 years old. All patients met the diagnostic criteria of COPD established by Respiratory Branch of Chinese Medicine Academy [4]. Patients who can't cooperate with treatment, had a history of allergy of glucocorticoid and treated with hormone recently were excluded. 110 cases of patients were divided into control group and observation group, 55 cases in each group. According to the statistical analysis, the differences in general condition and examination indexes of pulmonary function between two groups were not statistically significant and the results were comparable $(p>0.05)$.

\subsection{Methods of treatment}

All patients are routinely treated with anti-infection, oxygen inhalation, relieving cough, relieving sputum, correcting the balance of water-electrolyte, bronchodilator, etc. $2 \mathrm{~mL}$ of budesonide suspension (trade name: Pulmicort Respules, AstraZeneca in Sweden) was given to observation group on the base of routine treatment, the drug was taken by aerosol inhalation after imbedding atomizer, one time every 15 minutes, twice a day and 5-7 days for a course. The control group is treated with methylprednisolone intravenous therapy on the basis of routine treatment, $40-80 \mathrm{mg} /$ day and 5-7 days for a course. In the treatment period, we should observe the patients whether they have adverse reaction (including clinical adverse reactions and laboratory abnormalities) and take appropriate measures in time.

\subsection{Efficiency standard}

Marked effect: within three days after treatment, cough and expectoration of patients are obviously relieved, dyspnea, ralc and wheezing sounds in lungs disappear, hemogram is normal; effective: within three days after treatment, cough of patients is obviously relieved, the sputum volume is reduced slightly, the dyspnea relieves, the ralc and wheezing sounds in lungs reduces and their hemogram improves, but not recoveries normally; ineffective; Three days after treatment, the symptom and times of cough have no improvement or aggravate, the sputum volume is unchanged or increased, the dyspnea has no improvement or aggravate, the ralc and wheezing sounds in lungs have no improvement or aggravate and the hemogram has no improvement or increase. The marked effective rate and effective rate are calculated as the total efficiency [5].

\subsection{Statistics method}

Choosing the SPSS 13.0 statistical software to analyze, $p<$ 0.05 , the difference has statistical significance.

\section{Results}

\subsection{Comparison of clinical effect between two groups}

The clinical total efficiency of observation group is higher than the control group. According to the statistical analysis, the difference of efficacy between control group and observation group was significant $(p<0.05)$. The clinical effect of observation group is better. See Table 1.

\begin{tabular}{|c|c|c|c|c|c|c|}
\hline Group & $n$ & & $\begin{array}{l}\text { Marked } \\
\text { effect }\end{array}$ & Effective & Ineffective & $\begin{array}{c}\text { Total } \\
\text { efficiency } \\
(\%)\end{array}$ \\
\hline $\begin{array}{l}\text { Observation } \\
\text { group }\end{array}$ & 55 & 37 & $(67.3)$ & 13 (23.6) & $5 \quad(9.0)$ & 91.0 \\
\hline $\begin{array}{l}\text { Control } \\
\text { group }\end{array}$ & 55 & 23 & (41.8) & $20 \quad(36.4)$ & $12(21.8)$ & 78.2 \\
\hline
\end{tabular}

\subsection{Adverse reaction of two groups}

Among the patients of observation group who adopted aerosol inhalation of budesonide to treat acute exacerbation of COPD on the basis of routine treatment, there was one case of hoarseness and one case of pharyngodynia. After symptomatic treatment, the symptom has disappeared and there was no case of increased blood glucose.. As for the control group treated with methylprednisolone intravenous therapy on the basis of routine treatment, there were two cases of oral cavity fungus infection, eight cases of high blood glucose and one case of high blood pressure. After symptomatic treatment, all the symptoms has disappeared. According to the statistical analysis, the difference in the occurrence rate of adverse reaction between two groups has statistical significance $(p<0.05)$. The occurrence rate of adverse reaction of the observation group is significantly lower than the control group.

\section{Discussions}

COPD is a chronic airway inflammation disease. Related reports show that the mortality rate of patients who suffer from acute exacerbation of COPD has reached to $11 \%$ in the hospitalization period [6]. In clinical, the main methods for treatment of acute exacerbation of COPD are anti-inflammatory, oxygen inhalation, relieving cough and so on. In addition, adding glucocorticoid can reduce the inflammatory response, expand the bronchial and furthermore improve the efficacy. But the disadvantage of using glucocorticoid is more adverse reaction and low security.

The hormone inhaled therapy has small dosage. The drug has a direct effect on target organs without blood circulation. Therefore, it has good effect and less side effects. Budesonide is a middle efficiency inhaled adrenal cortical hormone. Its suspension is used for treatment of asthma attack in the past. The aerosol inhalation of budesonide can reach the whole lung of patients quickly, inhibit the hyperactivity of airway, thus reduce the secretion of gland and inhibit the production of mast cells [7]. The results of multicenter clinical study show that short-term aerosol inhalation of budesonide can significantly improve 
the clinical symptoms and lung function index of patients with acute exacerbation of COPD. Comparing with systemic hormone therapy, the efficacy is the same but the incidence of side effects decreases [8]. The results of this study show that the clinical effect of observation group is better than the control group and their incidence of adverse reaction is significantly lower than the control group. It fully reflects the clinical advantages of aerosol inhalation of budesonide in treatment of acute exacerbation of COPD. Meanwhile, it also shows the disadvantages of systemic hormone therapy. In a word, the aerosol inhalation of budesonide in treatment of acute exacerbation of COPD has defined effect, less adverse reaction and high security, worth of widely application in clinical.

\section{Reference:}

1. Cristina RR, Francisco JGT, Francisco JG, Jose M, Marin T. Classification of Chronic Obstructive Pulmonary Disease Severity According to the New Global Initiative for Chronic Obstructive Lung Disease 2011 Guidelines: COPD Assessment Test Versus Modified Medical Research Council Scale. Archivos de Bronconeumologia (English Edition). 2013.

2. Luo ZP, Zhou Y. clinical observation on aerosol inhalation of budesonide in treatment of Acute exacerbation of COPD. China Healthcare Innovation. 2010,5(13):22-23.
3. National Heart, Lung, and Blood Institute.Global initiative for chronic obstructive lung disease. NHLBI Workshop Report. 2003:Chapter4.

4. The chronic obstructive pulmonary disease group of Respiratory Branch of Chinese Medicine Academy. The guide on diagnosis and treatment of chronic obstructive pulmonary disease (Revised in 2007). Chinese Journal of Tuberculosis and Respiratory Diseases. 2007;30(1):8-17.

5. Wu TX. The efficacy of aerosol inhalation of budesonide in treatment of acute exacerbation of COPD. Clinical Education of General Practice. 2006;4(6):512.

6. Stanislaw D, Irena P, Aneta K, Adam Z, Wojciech B, Konrad P, Ivana S, Michal D, Anna K, Renata J, Piotr M. Met abolomics provide new insights on lung cancers tagging and discrimination from chronic obstructive pulmonary disease. Journal of Pharmaceutical and Biomedical Analysis. 2014;100.

7. Ye SL, Li CH. Clinical observation on the aerosol inhalation of budesonide in treatment of acute exacerbation of COPD.Journal of Jianghan University (Natural Science Edition). 2011;9(1):80.

8. Vera W, Christine S, Verena G, Michael S, Regina P, Claudia B, IreneJ H, Raymond V, Lena H, Steffen TS. Breathlessness, Functional Status, Distress and Palliative Care Needs Over Time in Patients with Advanced Chronic Obstructive Pulmonary Diseaseor Lung Cancer: A Cohort Study. Journal of Pain and Symptom Management. 2013. 